\title{
Diagnostic Value of Eosinopenia and Neutrophil to Lymphocyte Ratio in Early Onset Neonatal Sepsis
}

\author{
Ahmed M Abdelmoktader ${ }^{1}$, Sherin K Hussein ${ }^{2}$, Doaa Y Ali ${ }^{3}$,Ola M Eisa ${ }^{4}$ \\ 1. Professor of Pediatrics and Neonatology ,Faculty of Medicine, Fayoum University. \\ 2. Lecturer of pediatrics ,Faculty of Medicine, Fayoum University. \\ 3. Lecturer of clinical and chemical pathology,Faculty of Medicine, Fayoum University. \\ 4.M.B.B.CH , Faculty of Medicine, Fayoum University.
}

Corresponding author: prof . Ahmed M Abdelmoktader.

E-mail address: Abdelmoktader2006@yahoo.com.

Tel:01001802142

\begin{abstract}
:
Background: Neonatal sepsis remains a challenge for neonatal care providers. The accurate and timely diagnosis of neonatal sepsis remains a challenging issue due to its non-specific clinical presentation.
\end{abstract}

\section{Patients and Methods: This} descriptive cross sectional study was carried out at neonatal intensive care unit in Fayoum University Hospital from February to December 2019. Samples were obtained from 100 neonates that were met the inclusion criteria as having EONS. Neonates were subjected to: History taking, through clinical examination for manifestations of sepsis. Complete blood count, differential leucocytic count, Creactive protein and Blood culture were done to all neonates.

Results: of 100 neonates who met the inclusion criteria, $(61 \%)$ were males and (39\%) were females.71 (71\%) subjects were included in confirmed sepsis group and $29(29 \%)$ were in non-confirmed sepsis group. Mean eosinophil count in confirmed EONS and non- confirmed EONS group were $250 \pm 170$ cells $/ \mathrm{mm} 3$ and $670 \pm 470$ cells $/ \mathrm{mm} 3$, respectively, considered as significant $(\mathrm{p}<0.001)$. Result from diagnostic test of eosinopenia from the EONS group (cut-off point 280

cells $/ \mathrm{mm} 3$ ) obtained $56.3 \%$ sensitivity and $93.1 \%$ specificity. Mean NLR in EONS and non-confirmed EONS group were $3.08 \pm 1.9$ and $1.29 \pm 0.76$, respectively, also considered as significant $(p<0.001)$.Result for diagnostic test of NLR in EONS group (cut-off point 1.75) obtained $70.4 \%$ sensitivity and $75.9 \%$ specificity.

Conclusion: Eosinopenia and increased in NLR both have a high specificity value as a diagnostic marker of EONS.

Key words: Eosinophil count, Neutrophil to lymphocyte ratio, Early onset neonatal sepsis.

\section{Introduction:}

Neonatal sepsis is a clinical syndrome arising from the invasion of microorganisms into the blood stream that arises in the first month of life (1).Neonatal sepsis is a major cause of mortality in the developing countries. As the signs and symptoms of sepsis are nonspecific, early diagnosis poses a challenge to the clinicians (2). Neonatal sepsis is still a major problem in neonatal care and still 
contributes significantly to disability and death. At least 1 million deaths occuring in the newborn period (0-28 days) per year are caused by infection, of which nearly $25 \%$ is the cause of newborn mortality and accounting for $10 \%$ of infant mortality worldwide (3).

Evidence of infection from blood cultures itself often show insignificant results and takes too much time to obtain (4).Therefore, other test, such as a complete blood count (CBC) is done along with the blood culture to determine sepsis. However even with current severity and biomarkers which are derived from complete blood count, predicting outcomes of neonatal sepsis is still challenging. Conventional screening tests such as total and differential leukocyte counts, band cells, neutrophil counts and rapid immunological tests like C-reactive protein (CRP) assays may help in the diagnosis of septicemias; however, they lack the capacity to predict the severity of sepsis (5).

One of the important aspects of acute infection is the decline in eosinophil count that spreads through the blood circulation quickly and persistently (6).Efforts have been made to diagnose EONS using an easy and cheap tools such as eosinophil count and neutrophil to lymphocyte ratio (NLR) (7).

From the previous studies, eosinopenia has a good sensitivity and specificity in diagnosing sepsis on both EONS and LONS, but they were using adult's sepsis cut off point (7).

Moreover, bacterial products and cytokines released during sepsis also delay neutrophil apoptosis, which contributes to the degree of sepsis severity (8).

In this study, we tried to use point of intersection from neonatal subjects. The diagnostic value of NLR in EONS alone has never been done in neonates in Egypt.
This study were purposed to provide information about diagnostic value of eosinophil count and NLR for detecting EONS.

Patients and Methods: The current study was a descriptive cross sectional study that conducted in the neonatal intensive care unit (NICU) in Fayoum University Hospital and Itsa Central Hospital from February to December 2019. Study subjects were all neonates who met the inclusion criteria. We used consecutive sampling method with a sample size of 100 neonates. Detailed history and meticulous general \& systemic examinations were done, and also laboratory examination (hematology profile, differential count, eosinophil count, and blood culture).

\section{Inclusion criteria:}

1. Age (1-72 hours).

2. Any mode of delivery (vaginal or CS).

3. Both genders.

4. Sepsis clinical presentation (according to griffin score).

5. Sepsis risk factors: 2 major or 1 major and 2 or more minor

\section{Exclusion criteria:}

1-Any major congenital anomalies.

2-Any major surgical conditions.

3-Late onset neonatal sepsis (After 72 hours).

4- Healthy infants (term or preterm).

\section{Statistical Methods}

The collected data was organized, tabulated and statistically analyzed using SPSS software statistical computer package version 18 (SPSS Inc, USA).

\section{$\square$ For quantitative data:}

The mean, standard deviation (SD) were calculated. Independent t-test was used as a test of significance.

\section{$\square$ For qualitative data:}


The number and percent distribution was calculated and Chi-squared test was used as a test of significance.

$\square$ For interpretation of results of tests of significance, significance was adopted at $\mathrm{P}$ $\leq 0.05$.

\section{Ethical Consideration}

The study was reviewed by the Faculty of Medicine Research Ethical Committee.The participants were informed by the researcher about the objectives of the study, the examination, investigations that were done. Also the confidentiality of their information was respected and their right not to participate in the study was ensured.

\section{Results:}

There were 100 neonates suspected with sepsis that fulfill the inclusion criteria as in figure 1.

Incidence of EONS were mostly found in males, 61 (61\%), compared with females, $39(39 \%)$.

Blood culture were made and resulting in 37 positive growth, consist of klebsiella (16), MRSA (7), E.coli (4), staphylococcus aureus (3), CONS (3), gram negative bacilli (3) and acinobacter (1) .

Mean eosinophil count from the EONS group was $250 \pm 170$ cells $/ \mathrm{mm} 3$, meanwhile from non-EONS group was $670 \pm 470$ cells $/ \mathrm{mm} 3$ with $\mathrm{p}<0.001$ considered as significant. We also tallied their NLR and found that mean NLR from EONS and non-EONS group was $3.08 \pm$ 1.9 and $1.29 \pm 0.76$, respectively, with $\mathrm{p}<0.001$ considered as significant also. Figure 2A describes data distribution of eosinophil count in EONS and non-EONS group, while figure 4 describes data distribution of NLR in both groups.

An ROC curve to analyze the ability of eosinophil count to diagnose EONS (Fig.5) demonstrates that with a cut-off of 280 cells $/ \mathrm{mm} 3$, there is a $56.3 \%$ sensitivity and a $93.1 \%$ specificity,also the positive predictive value (PPV) was ( $96.8 \%$ ) and the negative predictive value (NPV) was $(36.2 \%)$.

A similar analysis with NLR showed that the best specificity and sensitivity for EONS was at NLR 1.75 with sensitivity of $70.4 \%$ and specificity $75.9 \%$, also the positive predictive value (PPV) was ( $91.7 \%$ ) and the negative predictive value (NPV) was( $40.5 \%$ )

\section{Discussion:}

In our study, neonates suspected with sepsis were found more in males than females. There were $\mathbf{1 0 0}$ neonates, in whom $61(61 \%)$ were males, $55 \%$ of cases were preterm with average gestational age $34.6 \pm 3$ weeks, average weight was $2.1 \pm$ $0.7 \mathrm{~kg}$, According to mode of delivery, 76 $\%$ of neonates suspected with sepsis were delivered by caesarean section, compared to $24 \%$ of neonates that were delivered normally, $37 \%$ of cases were culture proven cases of neonatal sepsis.

These findings were in accordance with Yogeeta et al (9), out of 150 clinically suspected neonates, $44(29.3 \%)$ were culture proven cases of neonatal sepsis. The males $(70.4 \%)$ were predominantly affected. Among neonates with EONS, 93.1\% were low birth weight and $86.3 \%$ were preterm. According to mode of delivery, $52.2 \%$ of neonates with sepsis were delivered by caesarean section, compared to $47.7 \%$ of neonates that were delivered normally.

This study showed that sepsis risk factors for the selected cases, $57 \%$ of mothers with premature rupture of membranes (PROM) $>18$ hours, $42 \%$ with history of intrapartum maternal fever, $3 \%$ with history of chorioamnionitis, $8 \%$ of cases were one of twins or triple, $34 \%$ of mothers were having urinary tract infection(UTI) and $41 \%$ with vaginal infections. 
Yogeeta et al (9), Study of maternal and neonatal risk factors showed significant association with premature rupture of membranes, $(\mathrm{p}$ value $=0.0001)$, maternal fever $(\mathrm{p}$ value $=0.01)$ and birth asphyxia $(\mathrm{p}$ value $=0.007$ ) with neonatal sepsis by using multivariate analysis.

We should have at least 2 clinical abnormal presentations, so these were the clinical presentations of our cases (10).

About $91 \%$ of cases presented with respiratory distress and need increase oxygen requirement, $\mathbf{8 9} \%$ with poor suckling and $\mathbf{6 0} \%$ with poor reflexes, $2 \%$ with convulsions and $4 \%$ were having (bradycardia ,apnea ,fever and hypothermia).

On the other hand, Velaphi et al,(11) reported in their study that the most common signs of sepsis included tachypnea (32\%), metabolic acidosis $(36 \%)$, lethargy $(15 \%)$, and hypothermia $(15 \%)$; whilst fever was uncommon $(0.6 \%)$.

Laboratory investigations were done to all cases to confirm or exclude EONS in the form of :

Complete blood picture with differential.

$>\mathrm{I} / \mathrm{T}$ ratio.

$>$ C-reactive protein.

$>$ Blood culture.

If there were at least 2 laboratory changes (leukocytosis or leucopenia, thrombocytopenia, I/T ratio $\geq 0.2$ ) with or without positive blood culture so the case was confirmed EONS (10).

Regarding blood culture in our study, $\mathbf{3 7 \%}$ of cases showed positive blood culture. This comes in agreement with Ferrieri et $\boldsymbol{a l},(12)$ study showing that positive blood culture only found in $30-40 \%$ sepsis cases. Complete blood count was done to all cases and showed that $39 \%$ of cases were having thrombocytopenia $(<100000), 52 \%$ of cases with leukopenia $(<5000)$ or leukocytosis (>20000), and increased I/T ratio $(\geq 0.2)$ in $22 \%$ of cases .

This was in agreement with, Medhat et $\boldsymbol{a l},(13)$ case control study that revealed: WBCs were statistically significantly $(\mathrm{P}=$ 0.030) higher among cases compared to normal controls, as the vast majority of controls had normal WBCs count (96.7\%), while only one case had leucocytosis (3.3\%). Among cases leucycytosis was seen in (48\%) and leucopenia was seen in (4\%).Moreover, platelet count showed a statistically significant $(\mathrm{P}<0.001)$ difference between cases and controls, with many of cases showing low platelet count. All of the controls had a normal PLT count, while $30 \%$ of cases had thrombocytopenia, the difference was statistically significant $(\mathrm{P}=0.001)$.

Regarding other markers of diagnosing sepsis, CRP level was elevated in $85 \%$ of cases in this study .This finding was in agreement with Sidra et al (14) who found that the mean CRP level was significantly higher in patients with sepsis than controls. Our cases were classified into 2 groups: confirmed sepsis (71\%) and nonconfirmed sepsis group (29\%), to compare eosinophils and NLR between the 2 groups.

In this study, mean eosinophil count was $0.25 \pm 0.17 \mathrm{k} /$ microliter $=250 \quad \pm 170$ cells/mm3 from the EONS group and as for non-confirmed EONS group it was $0.67 \pm 0.47 \mathrm{k} /$ microliter $=670 \pm 470$ cells/mm3 . Based on the logistic regression analysis, a very significant correlation was found between eosinophil count and sepsis occurrence with $\mathrm{p}$ value $(<\mathbf{0 . 0 0 0 1}(\mathbf{S}))$, also the positive predictive value (PPV) was $96.8 \%$ and the negative predictive value (NPV) was $36.2 \%$.

The ROC analysis showed AUC 80.8\% with an eosinophil cut-off point of 280 cells $/ \mathrm{mm} 3$ and sensitivity $=56.3 \%$, specificity $=93.1 \%$. 
Our results were in agreement with Wilar,(15) who reported that mean eosinophil count was $169.8 \pm 197.1$ cells/mm3 from the EONS group and as for non-EONS group it was $405.7 \pm 288.9$ cells $/ \mathrm{mm} 3$. Based on the logistic regression analysis, a very significant correlation was found between eosinophil count and sepsis occurrence with $(p<0.001)$, where the lower the eosinophil count is, the higher the chance of sepsis to take place.

Our results were in concordance with those obtained by Bagus et al, (16) who conducted a study to compare the diagnostic values of immature granulocytes, eosinopenia and IT ratios in detecting EONS in neonates (0-6 hours) with the risk of bacterial infection, where as eosinopenia showed the highest specificity of detection to detect EONS.

This study also looking for NLR corellation with EONS, and we found that mean NLR was 3.08 \pm 1.9 from the EONS group and 1.29 \pm 0.76 for non-EONS group. Based on the logistic regression analysis, a very significant correlation also found between NLR and sepsis incidence, where the higher the ratio, the higher the incidence of EONS as well with $\mathrm{p}$ value $(<0.0001)$, also the positive predictive value (PPV) was $91.7 \%$ and the negative predictive value (NPV) was $40.5 \%$.

Based on the ROC curve with a 1.75 cutoff point, the result shows $70.4 \%$ sensitivity, $75.9 \%$ specificity.

Our results were in agreement with Wilar,(15) who found that the mean NLR from the EONS and the non EONS group was $2.82 \pm 2.29$ and $0.82 \pm 0.32$, respectively, also with $(\mathrm{P}<0.001)$ considered as significant.

A similar analysis with NLR showed that the best specificity and sensitivity for EONS was at NLR 1.245 with sensitivity of $83.3 \%$ and specificity $93.3 \%$, and these results are near to our results.

On the other hand Dursun et al,(17) reported that NLR had a sensitivity of $75.6 \%$, a specificity of $38.4 \%$, a PPV of $35.6 \%$, and a NPV of $77.8 \%$ to predict sepsis in children.

Yu et al,(18) reported that multiple studies have supported the role of the neutrophilto-lymphocyte ratio ( NLR) as a prognostic tool, demonstrating worse outcomes with higher NLR values.

Limitations of this study is there was no follow-up examination in patients with higher levels of

eosinophil counts and low NLR. To determine the risk of mortality in neonates with neonatal sepsis,

serial examination of eosinophil and NLR is required.

\section{Conclusion:}

From our study we concluded that eosinopenia and an increased in neutrophil to lymphocyte ratio (NLR) both have a high specificity value as a diagnostic marker of EONS.

\section{References:}

(1) Rusell $A B$ and Isaacs D: Infection in the newborn. In: Rennie JM, Robertson NR, editors.Rennie \& Roberton's textbook of neonatology. 5th ed. London: Churchill Livingstone 2012.p.101331.

(2) Snehal L. Martin, Saumil Desaib, Ruchi Nanavatib, Roshan B.Colaha, Kanjaksha Ghosha andMalay B. Mukherjeea.: Red cell distribution width and its association with mortality in neonatal sepsis. The Journal Of Maternal -Fetal \& Neonatal Medicine 2018.

(3) Makkar M, Gupta C, Pathak R, Garg S, Mahajan NC. Performance 
evaluation of hematologic scoring system in early diagnosis of neonatal sepsis. J Clin Neo 2013:25-9.

(4) Kayange N, Kamugisha E, Mwizamholya DL, Jeremiah S, Mshana SE : Predictors of positive blood culture and deaths among neonates with suspected neonatal sepsis in tertiary hospital, MwanzaTanzania. BMC Pediatrics 2010;10:1-9.

(5) Jajoo M, Kapoor K, Garg LK., et al.: To study the incidence and risk factors of early onset neonatal sepsis in an out born neonatal intensive care unit of India. J Clin Neonatal. 2015;4:91-95.

(6) El-Din, El- Sokkary, Bassiouny, Hassan. Epidemiology of Neonatal Sepsis and Implicated Pathogens: A Study from Egypt. 2015. Biomed Research International 2015: 509484 .

(7) Shaaban H, Daniel S, Sison R, Slim J, Perez G. Eosinopenia: is it a good marker of sepsis in comparison to procalcitonin and c-reactive protein levels for patient admitted to a critical care unit in an urban hospital?. J Crit Care 2010;25:5705.

(8) Steams-Kurosawa DJ, Osuchowsky MF, Valentine C, Kurosawa S, Remick DG. The pathogenesis of sepsis. Annu Rev Pathol 2011;6:1948.

(9) Yogeeta Bala,V.S. Randhawa1, Arvind Saili2, Ravinder Kaur1,Shweta Chitkara1 and Aditya Duggal3 Indian Journal of Applied Microbiology July - September 2018, pp. 20-28.

(10) Shrestha S, Dongol Singh S, Shresta NC, Shrestha RPB, Madhup SK. Comparison of clinical and laboratory parameters in culture proven and unproven early onset sepsis in NICU. Kathmandu Univ Med J 2013;44(4):310-14.

(11) Velaphi SC, Westercamp M, Moleleki M, Pondo T, Dangor Z, Wolter N, et al. (2019) Surveillance for incidence and etiology of earlyonset neonatal sepsis in Soweto, South Africa. PLoS ONE 14(4): e0214077.

(12) Ferrieri P, Wallen LD. Neonatal bacterial sepsis. In: Gleason CA, Devaskar SU, editors. Avery's disease of the newborn 9th . Philadephia. Saunders Elsevier 2012:538-50.

(13) Medhat A Saleh, Yasser T Kasem, Hesham H Amin.: Evaluation of neonatal sepsis and assessment of its severity by Red Cell Distribution Width indicator. The Egyptian Journal of Community Medicine Vol. 35 No. 3 July 2017.

(14) Sidra Younis, Muhammad Ali Sheikh, Amjad Ali Raza: Diagnostic Accuracy of C - reactive protein in Neonatal Sepsis. J. Bio resource Manage 2014. 1(1): 33-42.

(15) Wilar R. Diagnostic Value of Eosinopenia and Neutrophil to Lymphocyte Ratio on Early Onset Neonatal Sepsis Korean J Pediatr. 2018.

(16) Bagus E, Kahar H, Wardhani P. Diagnostic values of immature granulocytes, eosinopenia and I/T ratio in detection of early onset neonatal sepsis in neonates with bacterial infection risk. Folia Medika Indonesina 2014;50:43-7.

(17) Dursun A, Ozsoylu S, Akyildiz BN. Neutrophil-to-lymphocyte ratio and mean platelet volume can be useful markers to predict sepsis in 
children. Pak J Med Sci 2018;34:918-22.

(18) Y. Yu, H. Wang, A. Yan, H. Wang, X. Li, J. Liu, W. Li, Pretreatment neutrophil to lymphocyte ratio in determining the prognosis of head and neck cancer: a metaanalysis, BMC Cancer 18 (1) (2018) 383. 


\section{Figure (1): Inclusion criteria:}

\section{Inclusion criteria:}

- Age 1-72 hours.

- Sepsis risk factors: 2 major or 1 major and 2 or more minor Major include: PROM > 18 hours, intrapartum fever $>38 \mathrm{C}$, chorioamnionitis, greenish and foul smelling amniotic fluid, and fetal heart rate $>160 \mathrm{bpm}$. Minor include: PROM > 12 hours, intrapartum fever $>37.5 \mathrm{C}$, low apgar score, very low birth weight, vaginal discharge untreated, and untreated UTI.

- Sepsis clinical presentation (griffin score).

Neonates suspected as having early onset neonatal sepsis (EONS) according to griffin score, $n=100$

- Signing informed consent

- Draw blood for Septic Work Up Examination, positive if $\geq 2$

o Complete blood count (leucopenia or leucocytosis, thrombocytopenia) o ITR $>0.2$

o $C R P>6 \mathrm{mg} / \mathrm{L}$

o With positive or negative blood culture (Shrestha et al,.2013).

c(71) confirmed EONS:

- $(+)$ risk factors

- (+) sepsis clinical presentation

- (+) septic work up
(29) Non-confirmed EONS:

- (+) risk factors

- (+) sepsis clinical presentation

- (-)septic work up septic work up

- Eosinophil count

Data analysis:

- Neutrophil to lymphocyte ratio (NLR) 


\section{Figure (2):Proportion of Confirmed}

\section{Sepsis and Non Confirmed Sepsis Groups}

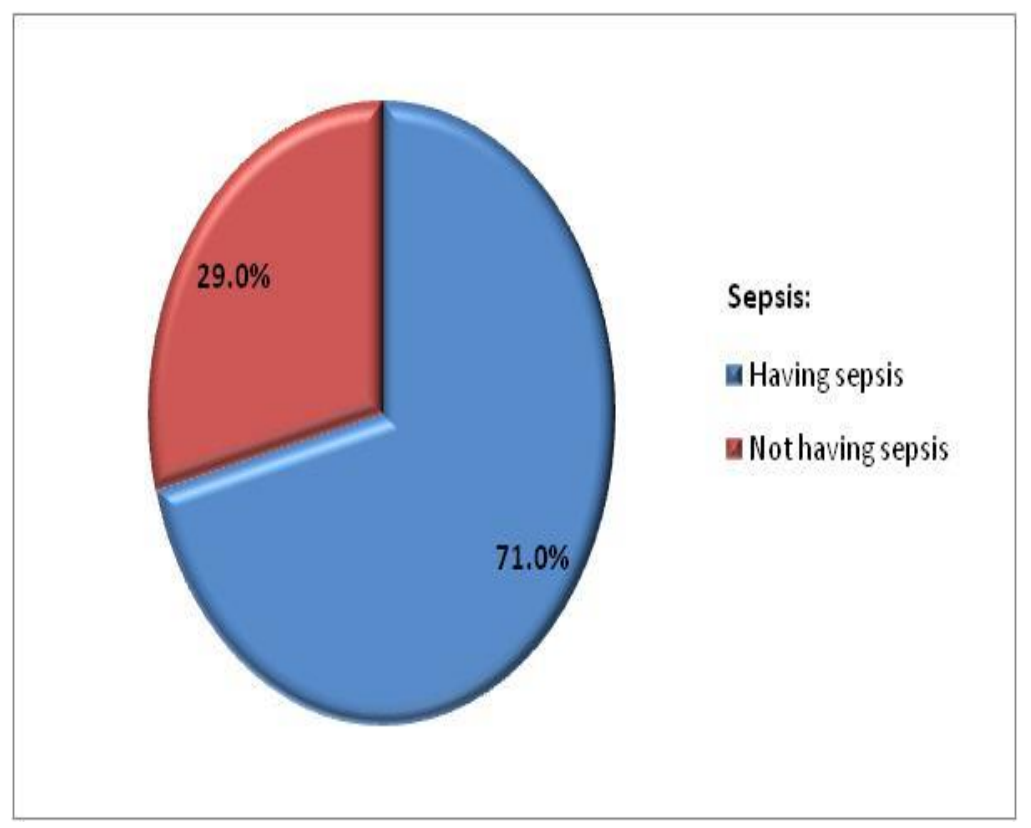




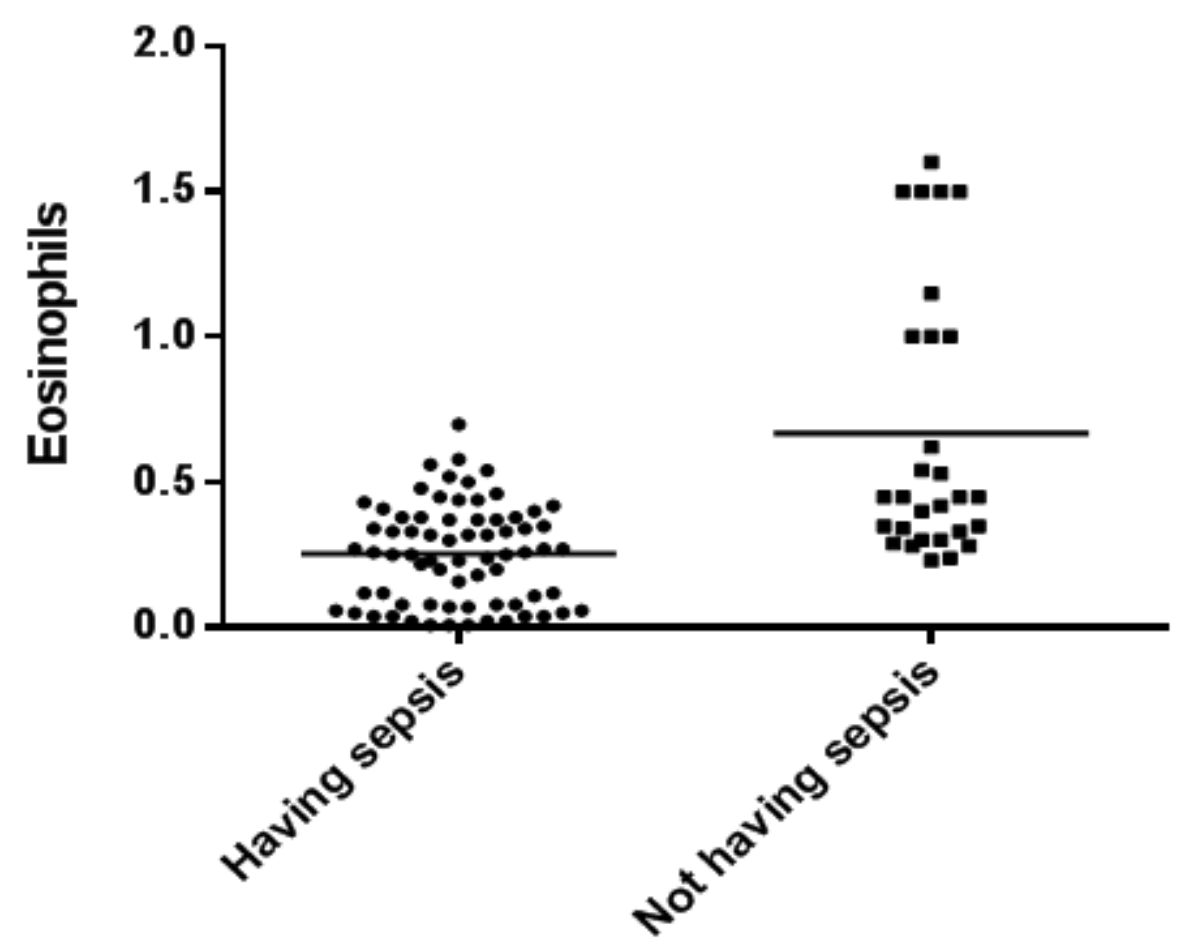

Figure (3): Proportion of Eosinophils Among the Study Groups

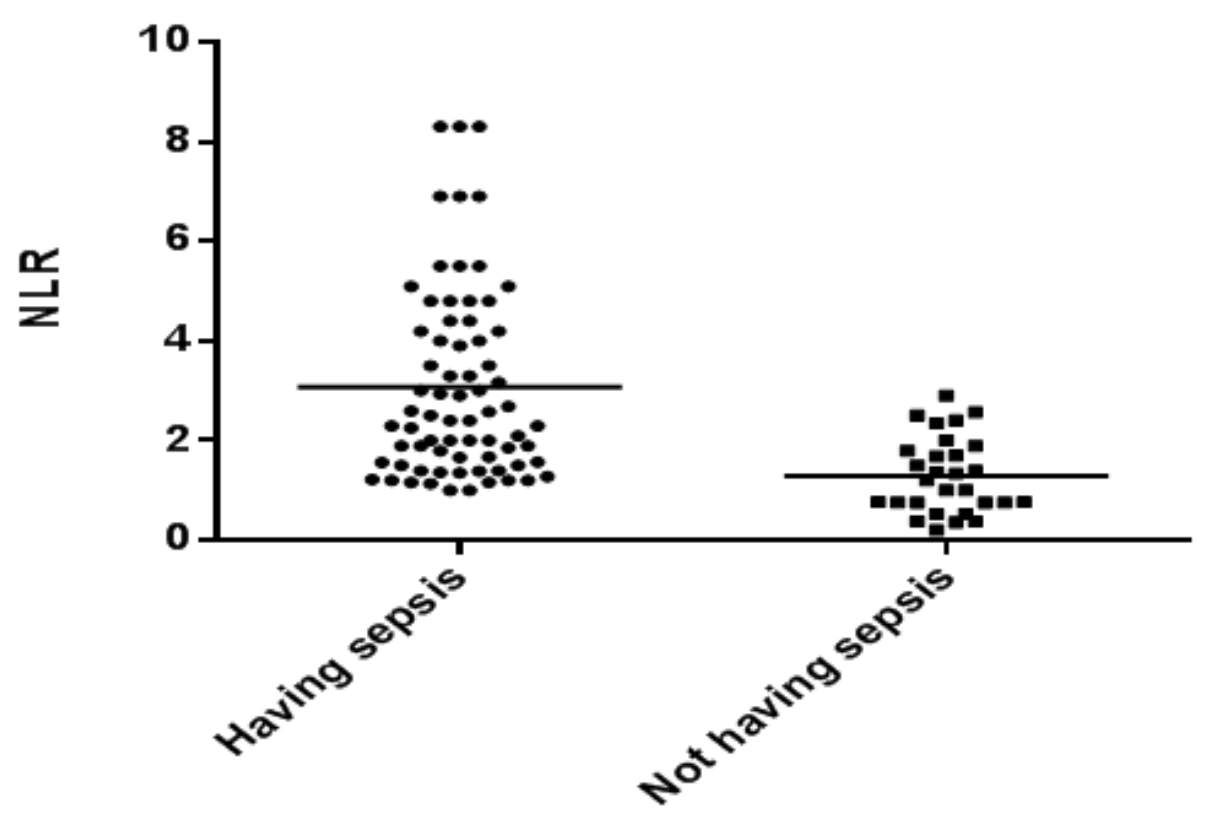

Figure (4):Proportion of NLR Among the Study Groups 


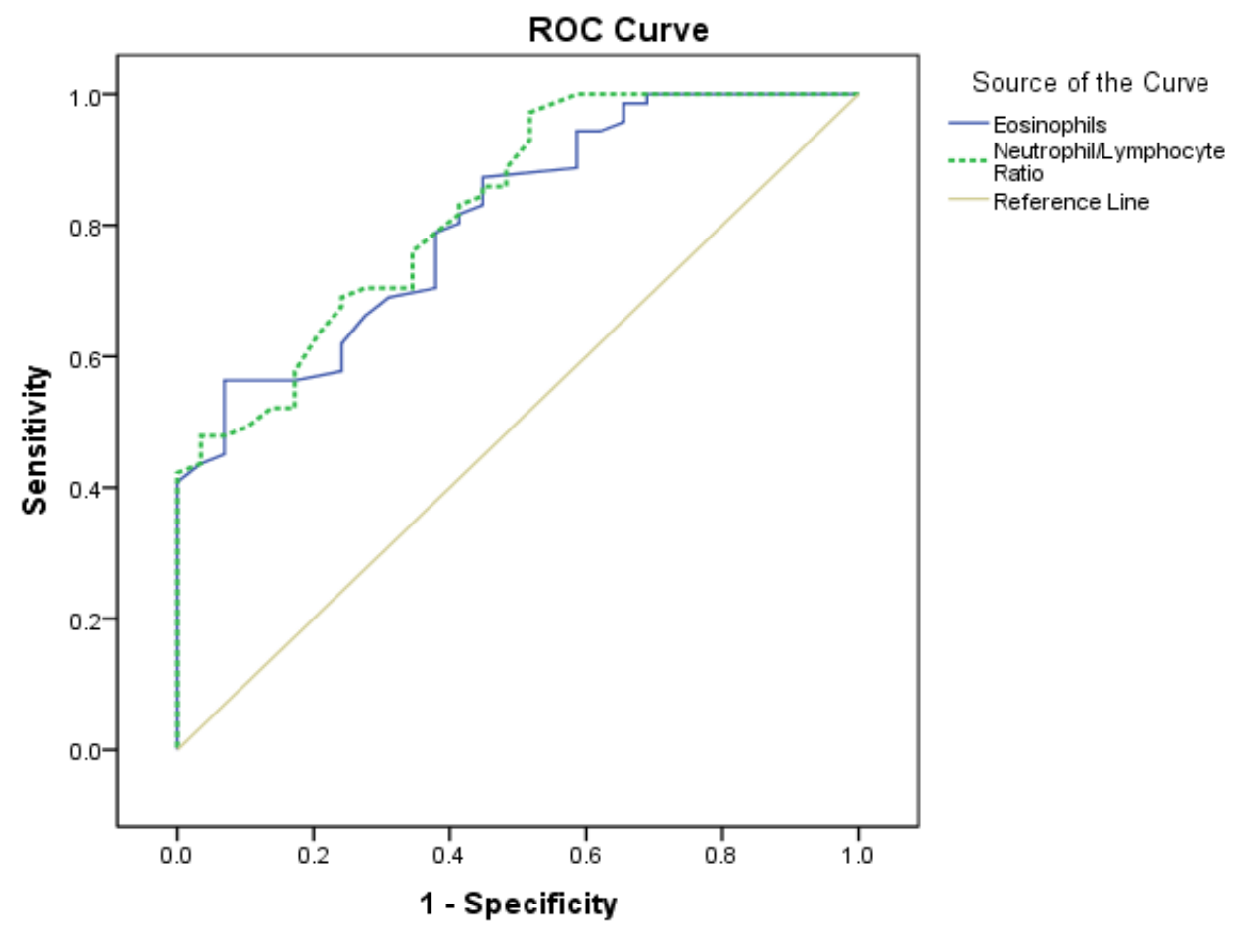

Diagonal segments are produced by ties.

Figure (5):ROC Curve for Detecting EONS 